\title{
Téoros
}

Revue de recherche en tourisme

\section{L’Orient, géographie imaginaire}

\section{Les écrivains français et les villes de désir}

\section{Martine Geronimi}

Volume 25, numéro 2, été 2006

Désirs d’Orient : du passé vers l'avenir

URI : https://id.erudit.org/iderudit/1071062ar

DOI : https://doi.org/10.7202/1071062ar

Aller au sommaire du numéro

Éditeur(s)

Université du Québec à Montréal

ISSN

0712-8657 (imprimé)

1923-2705 (numérique)

Découvrir la revue

Citer cet article

Geronimi, M. (2006). L’Orient, géographie imaginaire : les écrivains français et les villes de désir. Téoros, 25(2), 13-18. https://doi.org/10.7202/1071062ar d'utilisation que vous pouvez consulter en ligne.

https://apropos.erudit.org/fr/usagers/politique-dutilisation/ 


\section{L'Orient, géographie imaginaire Les écrivains français et les villes de désir}

\section{Martine Geronimi}

On ne peut parler de l'Orient et de sa géographie imaginaire sans aborder l'œuvre capitale du Français Antoine Galland, véritable passeur interculturel en tant que premier traducteur du Coran et du récit fondateur Les Mille et Une Nuits. Cet érudit de la fin du $X V \|^{e}$ siècle a voulu faire connaître à ses contemporains les philosophies orientales. Ce voyageur, qui parcourt pendant quinze ans l'Orient méditerranéen au travers de voyages "de travail », a eu le mérite d'avoir ouvert l'horizon des hommes des siècles subséquents sur le faste d'un Orient moderne, dont il essaiera de transmettre dans Les Mille et Une Nuits les éléments les plus remarquables. L'Orient qu'il décrit est un Orient merveilleux. L'émotion esthétique qu'il a ressentie lors de ses voyages s'est combinée au choc des découvertes liées à ses pérégrinations érudites. C'est en cherchant les villes disparues et la civilisation démantelée de la Grèce antique que ce voyageur a découvert un autre monde, celui de l'Orient.

On ne peut mettre en doute que les voluptés associées aux nuits orientales décrites dans ce récit n'aient donné le goût aux hommes du XIX de tenter le voyage en Orient. II est reconnu que l'expression "Voyage en Orient » a été forgée par les romantiques. Cet espace investi d'imaginaire associé à un lieu de mémoire devient alors un espace touristique au sens contemporain, espace dont les contours bien que flous ont été transformés en objet de désir par les écrivains doués d'une belle plume. Ces romantiques ont créé la soif d'aller connaitre, voire reconnaître, ces lieux foulés par des précurseurs et de combler cette page vide, laissant place à l'imagination la plus débridée.
Mais ce désir d'Orient s'exacerbe en France après 1798 et la campagne d'Égypte faite par Napoléon. On voit alors des écrivains en mal de voyage initiatique partir fouler les terres orientales; de Lamartine à Loti, les témoignages sont nombreux et fort intéressants.

Dans cet article, je vais m'attarder sur quelques grands écrivains français qui ont porté leur regard sur les villes orientales devenues objet de désir. Je m'intéresse à cette géographie imaginaire construite autour de ce désir d'Orient. Si la classe moyenne anglaise et américaine, à partir des premiers voyages à forfait du célèbre voyagiste $\mathrm{Cook}^{2}$, constitue la première vague de tourisme de masse à se rendre, elle aussi, en Orient ${ }^{3}$, des touristes de distinction français continuent à voyager en solitaire et à rapporter des récits de voyage agrémentés de photographies ou de dessins. Ils suivent les traces d'illustres précurseurs et sont sous l'emprise de deux œuvres majeures, l'Itinéraire de Paris à Jérusalem de Chateaubriand de 1806 et le Voyage en Orient de Lamartine de 1839 .

\section{Du dépaysement à la déterritorialisation, de l'exotisme à l'Ailleurs}

Jusqu'à la fin de la Première Guerre mondiale et depuis le début du XIX ${ }^{e}$ siècle, le voyage en Orient est un rite de passage pour les jeunes bourgeois français. Cet espace oriental se déploie autour des rives orientales de la Méditerranée. Constantinople est la ville centrale et aussi la porte de cet orient levantin, cette mosaïque où se mêlent et se côtoient l'islam et la chrétienté. Ce voyage initiatique conduit les jeunes pèlerins de Grèce en Turquie, de Syrie en Palestine, et se ter-

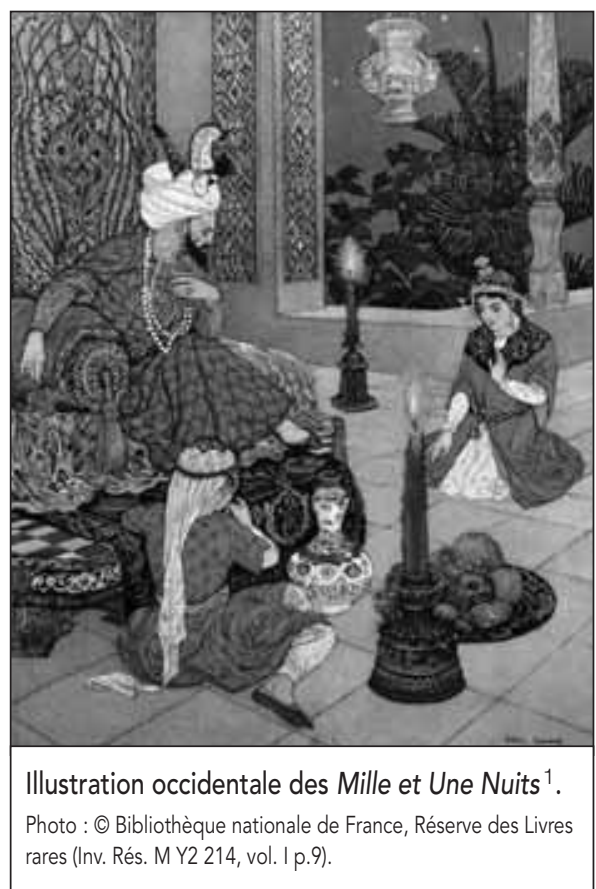

mine en Égypte. Le but du voyage est double: à la fois acquérir la connaissance et soutenir le désir. On peut parler de tourisme de distinction, mais on ne peut omettre que la vision de ces voyageurs reprend les vues de la colonisation sous une forme adoucie, mais tout de même très critique. La fascination du voyageur se lit dans ce périple symbolique qui reflète les visions d'un imaginaire collectif.

Les villes que nous allons parcourir au travers de la vision des écrivains voyageurs suivent l'itinéraire classique, inauguré en 1811 par Chateaubriand, un trajet autour de la mer Méditerranée (Égypte, Palestine, Liban, Constantinople). Ce parcours et cet espace littéraire représentent le voyage romantique par excellence. 


\section{Alexandrie, lieu de passage}

Alexandrie ne semble pas avoir connu un immense succès auprès des voyageurs. La raison en est simple, elle n'est pas conforme au rêve d'Orient tel que fabriqué avant le voyage par l'imaginaire des Français qui la visitent. Cette Alexandrie de rêve, celle du révéré Alexandre le Grand, n'existe pas. La ville est trop cosmopolite pour satisfaire le voyageur épris d'exotisme. Dès 1798, Alexandrie n'intéresse pas Bonaparte durant sa célèbre campagne d'Égypte et les 140 savants qui l'accompagnent ne trouvent pas ce qu'ils y cherchent, les traces d'Alexandre. Les orientalistes qui ont forgé au XVIII ${ }^{\mathrm{e}}$ siècle le goût des Français et en particulier celui de Bonaparte, comme Volney ou Venture de Paradis, ont créé un Orient idyllique. Le rêve de Bonaparte est aussi dicté par Talleyrand, son ministre: "L'Égypte fut une province de la République romaine, il faut qu'elle le devienne de la République française ${ }^{4}$. " Le goût pour l'Orient est aussi un goût de coIonisation. Mais la campagne d'Égypte se solde par un fiasco avec plus de 10000 morts, notamment de la peste.

Quand Chateaubriand s'y rend en 1806, il le fait très rapidement et montre dans son récit publié en 1811, Itinéraire de Paris à Alexandrie, combien il est désappointé par le lieu :

Si j'avais été enchanté de l'Égypte, Alexandrie me sembla le lieu le plus triste et le plus désolé de la terre. Du haut de la terrasse de la maison du consul, je n'apercevais qu'une mer nue, qui se brisait sur des côtes basses encore plus nues, des ports presque vides et le désert libyque s'enfonçant à l'horizon du midi; ce désert semblait, pour ainsi dire, accroitre et prolonger la surface jaune et aplanie des flots : on aurait cru voir une seule mer dont une moitié était agitée et bruyante, et dont l'autre moitié était immobile et silencieuse. Partout la nouvelle Alexandrie mêlant ses ruines aux ruines de l'ancienne cité; un Arabe galopant sur un âne au milieu des débris; quelques chiens maigres dévorant des carcasses de chameaux sur la grève; les pavillons des consuls européens flottant au-dessus de leurs demeures, et déployant, au milieu des tombeaux, des couleurs ennemies: tel était le spectacle [...]. Ces lieux sont d'autant plus tristes, que les Anglais ont noyé le vaste bassin qui servait comme de jardin à Alexandrie. L'œil ne rencontre plus que du sable, des eaux et l'éternelle colonne de Pompée.
En 1849, dans une lettre adressée à sa mère, Flaubert s'écrie: "Alexandrie, d'ailleurs, est presque un pays européen, tant il y a d'Européens. Nous sommes, à la table d'hôte de notre hôtel, une trentaine. Tout est plein d'Anglais, d'Italiens, etc. " Un peu plus loin, il explique avec déception: "C'est au Caire que l'Orient commence. Alexandrie est trop mélangée d'européens pour que la couleur locale y soit bien pure ».

Gérard de Nerval publie en 1867 une description d'Alexandrie découverte lors de son voyage de 1843 :

L'Égypte est un vaste tombeau; c'est l'impression qu'elle m'a faite en abordant sur cette plage d'Alexandrie, qui, avec ses ruines et ses monticules, offre aux yeux des tombeaux épars sur une terre de cendres.

Des ombres drapées de linceuls bleuâtres circulent parmi ces débris. Je suis allé voir la colonne de Pompée et les bains de Cléopâtre. La promenade du Mahmoudieh et ses palmiers toujours verts rappellent seuls la nature vivante [...].

Je ne parle pas d'une grande place toute européenne formée par les palais des consuls et par les maisons des banquiers, ni des églises byzantines ruinées, ni des constructions modernes du Pacha d'Égypte, accompagnées de jardins qui semblent des serres. J'aurais mieux aimé les souvenirs de l'antiquité grecque, mais tout cela est détruit, rasé, méconnaissable.

Ce ne sont donc pas ces voyageurs français qui établiront la réputation d'Alexandrie comme destination fascinante de voyage. II faut se tourner vers les Anglais Edward Morgan Forster et Lawrence Durell. Forster rédigea un guide et une histoire d'Alexandrie en 1922. Ces deux livres ont servi à toute une génération de voyageurs à la recherche de l'Alexandrie cosmopolite des années 1920. Durell, dans les années 1940, métamorphose cette ville en héroïne de ses romans (le quatuor d'Alexandrie). Enfin, le Grec Constantin Cavafy et l'Égyptien Naguib Mahfouz en font la ville des passions.

\section{Jérusalem, la cité de la désolation}

Quittant rapidement Alexandrie, nos voyageurs se rendaient en Palestine, notamment à Jérusalem. Tel Chateaubriand, l'écrivain voyageur accomplit un pèlerinage. II veut se retrouver sur les lieux saints. II veut voir le centre de la chrétienté, mais est aussi curieux de voir les cultures et les religions qui cohabitent dans l'espace restreint de la ville.

L'attente de la découverte semble provoquer plus d'émotion à Chateaubriand que le fait de se retrouver au milieu de la ville sainte. Son émotion est faite de crainte et de respect. II avoue même :

J'aperçus enfin moi-même cette montagne comme une tache ronde au-dessous des rayons du soleil. Je me mis alors à genoux à la manière des Latins. Je ne sentis point cette espèce de trouble que j'éprouvai en découvrant les côtes de la Grèce; mais la vue du berceau des Israélites et de la patrie des chrétiens me remplit de crainte et de respect.

Il est alors conscient de marcher sur les pas d'illustres prédécesseurs; il ressent l'héritage qui le relie aux croisés et s'exclame en citant Godefroy de Bouillon, Raimond de SaintGilles, Tancrède le Brave, Hugues le Grand, Richard Cœur de Lion et même Saint-Louis : "Obscur pèlerin, comment oserais-je fouler un sol consacré par tant de pèlerins illustres?"

Sa description de Jérusalem est ensuite digne du meilleur géographe par sa précision :

Vue de la montagne des Oliviers, de l'autre coté de la vallée de Josaphat, Jérusalem présente un plan incliné sur un sol qui descend du couchant au levant. Une muraille crénelée, fortifiée par des tours et par un château gothique, enferme la ville dans son entier, laissant toutefois au dehors une partie de la montagne de Sion, qu'elle embrassait autrefois.

Dans la région du couchant et au centre de la ville, vers le Calvaire, les maisons se serrent d'assez près; mais au levant, le long de la vallée de Cédron, on aperçoit des espaces vides, entre autres l'enceinte qui règne autour de la mosquée bâtie sur les débris du temple, et le terrain, presque abandonné, où s'élevaient le 
château Antonia et le second palais d'Hérode. Les maisons de Jérusalem sont de lourdes masses carrées, fort basses, sans cheminées et sans fenêtres; elles se terminent en terrasses aplaties ou en dômes, et elles ressemblent à des prisons ou à des sépulcres.

Toutefois l'écrivain romantique enjolive sa description lorsqu'il interprète le paysage et traduit ses sentiments :

Tout serait à l'œil d'un niveau égal, si les clochers des églises, les minarets des mosquées, les cimes de quelques cyprès et les buissons de nopals, ne rompaient l'uniformité du plan. À la vue de ces maisons de pierre, renfermées dans un paysage de pierres, on se demande si ce ne sont pas là les monuments confus d'un cimetière au milieu d'un désert.

Son sens critique s'exacerbe quand il dépeint la ville dans toute sa désolation:

Le paysage qui environne la ville est affreux; quelle désolation et quelle misère! Dans cet amas de décombres, qu'on appelle une ville, il a plu aux gens du pays de donner des noms de rues à des passages déserts. Les maisons ressemblent à des prisons ou à des sépulcres. À la vue de ces maisons de pierres, on se demande si ce ne sont pas là les monuments confus d'un cimetière au milieu d'un désert. Entrez dans la ville, rien ne vous consolera de la tristesse extérieure: vous vous égarez dans les petites rues non pavées, qui montent et descendent sur un sol inégal et vous marchez dans les flots de poussière, ou parmi des cailloux roulants.

Lamartine, dans son livre Le voyage en Orient de 1835, est beaucoup plus lyrique dans une phrase fleuve:

C'était elle! Elle se détachait en jaune sombre et mat, sur le fond bleu du firmament et sur le fond noir du mont des Oliviers. Nous arrêtâmes nos chevaux pour la contempler dans cette mystérieuse et éblouissante apparition. Chaque pas que nous avions à faire, en descendant dans les vallées profondes et sombres qui étaient sous nos pieds, allait de nouveau la dérober à nos yeux : derrière ces hautes murailles et ces dômes abaissés de Jérusalem, une haute et large colline s'élevait en seconde ligne, plus som- bre que celle qui portait et cachait la ville: cette seconde colline bordait et terminait pour nous l'horizon.

Cette ferveur et cette religiosité ne se retrouvent absolument pas chez Flaubert qui, en 1850, dépeint la ville avec une vue critique et très visionnaire. Dans une écriture étonnamment moderne, il ose désacraliser le lieu et le dépeindre dans la configuration la plus noire qui soit :

Jérusalem est un charnier entouré de murailles. Tout y pourrit, les chiens morts dans les rues, les religions dans les églises. Il y a quantité de merdes et de ruines ${ }^{5}$.

Le Saint-Sépulcre est l'agglomération de toutes les malédictions possible. Dans un si petit espace, il y a une église arménienne, une grecque, une latine, une copte. Tout cela s'injuriant, se maudissant du fond de l'âme, et empiétant sur le voisin à propos de chandeliers, de tapis et de tableaux, quels tableaux! C'est le pacha turc qui a les clefs du SaintSépulcre; quand on veut le visiter, il faut aller chercher les clefs chez lui. Je trouve ça très fort; du reste c'est par humanité. Si le Saint-Sépulcre était livré aux chrétiens, ils s'y massacreraient infailliblement. On en a vu des exemples.

Comme art, il n'y a rien que d'archipitoyable dans toutes les églises et couvents d'ici.

Flaubert n'apprécie aucunement cette ville et la quitte pour se rendre à Beyrouth.

\section{Beyrouth, la ville des voluptés}

Beyrouth encensé par Lamartine l'avait proprement ébloui :

La ville occupe une gracieuse colline qui descend en pente douce vers la mer; quelques bras de terre ou de rochers s'avancent dans les flots et portent des fortifications turques de l'effet le plus pittoresque; la rade est fermée par une langue de terre qui défend la mer des vents d'est; toute cette langue de terre, ainsi que les collines environnantes sont couvertes de la plus riche végétation; les mûriers à soie sont plantés partout et élevés d'étage en étage sur des terrasses artificielles; les caroubiers à la sombre verdure et au dôme majestueux.
Séduit par la végétation, cet écrivain romantique en fait les louanges:

Les figuiers, les platanes, les orangers, les grenadiers et une quantité d'autres arbres ou arbustes étrangers à nos climats, étendent sur toutes les parties du rivage, voisines de la mer, le voile harmonieux de leurs divers feuillages; plus loin, sur les premières pentes des montagnes, les forêts d'oliviers touchent le paysage de leur verdure grise et cendrée : à une lieue environ de la ville, les hautes montagnes des chaînes du Liban commencent à se dresser; elles y ouvrent des gorges profondes, où l'œil se perd dans les ténèbres du lointain; elles y versent de larges torrents, devenus des fleuves.

Pourtant, une fois de plus, Flaubert ne se plait pas dans cet Orient-là ; il n'apprécie pas ce lyrisme qui l'entoure et voudrait s'échapper du lieu oriental qui une fois de plus le déçoit. À tel point qu'il écrit, en juillet 1850, à son ami Frédéric Baudry : "Savez-vous, cher ami, quel sera quant à moi le résultat de mon voyage d'Orient? Ce sera de m'empêcher d'écrire jamais une seule ligne sur l'Orient. »

Or, à son arrivée, il semble séduit et écrit à sa mère :

En face de nous Beyrouth, avec ses maisons blanches, bâtie à mi-côte et descendant jusqu'au bord des flots, au milieu de la verdure des mûriers et des pins parasols. Puis, à gauche, le Liban, c'est-à-dire une chaîne de montagnes portant des villages dans les rides de ses vallons, couronnée de nuages et avec de la neige à son sommet.

Il faut avouer que Flaubert ne pense qu'à une seule chose: fréquenter les femmes de Beyrouth, celles qui vendent leurs charmes dans les maisons closes. Lui et son compagnon de voyage, Maxime du Camp, préfèrent passer leur temps dans les lupanars. II garde de Beyrouth d'ailleurs un fort mauvais souvenir, puisqu'il y contracte la vérole et s'en plaint à son ami Brouillet depuis Constantinople.

Cette vision des femmes rencontrées par les voyageurs arrivés à Beyrouth est totalement différente dans l'évocation romantique d'un Lamartine, lorsque celui-ci dépeint le bain des femmes ou d'un Nerval qui se voit offrir la possibilité de côtoyer les 
femmes d'un scheik maronite. Nerval semble également priser les mœurs libérées de Beyrouth, mais il apprécie et comprend particulièrement bien dans "son voyage en Orient » le rôle du voile que portent les femmes:

\section{Je n'avais pas compris tout d'abord ce qu'a d'attrayant ce mystère dont s'en- veloppe la plus intéressante moitié du peuple d'Orient; mais quelques jours ont suffi pour m'apprendre qu'une femme qui se sent remarquée trouve généralement le moyen de se laisser voir, si elle est belle. Celles qui ne le sont pas savent mieux maintenir leurs voiles, et l'on ne peut leur en vouloir. C'est bien là le pays des rêves et de l'illusion! La laideur est cachée comme un crime, et I'on peut toujours entrevoir quelque chose de ce qui est forme, grâce, jeu- nesse et beauté.}

Il comprend que cet élément de la tenue féminine n'est pas qu'un simple tissu, mais un phénomène majeur de la culture orientale. Nerval approche I'Orient avec une ouverture d'esprit que ne possède pas Flaubert au même moment. II s'ouvre à l'Autre.

\section{Constantinople, la porte des Mille et Une Nuits}

Si Lamartine avait adoré Beyrouth, sa vision de Constantinople est aussi exaltée: "Vous avez à toutes les heures du jour et de la nuit le plus magnifique et le plus délicieux spectacle dont puisse s'emparer un regard humain ; c'est une ivresse des yeux qui se communique à la pensée, un éblouissement du regard et de l'âme. 》

En revanche, Chateaubriand écrivait sa déception: "Le séjour de Constantinople me pesait. Je n'aime à visiter que les lieux embellis par les vertus et les Art, et je ne trouvais dans cette patrie de Phocas et de Bajazet ni les unes ni les autres. »

Ce qui fera dire à Maxime du Camp, compagnon de voyage de Flaubert:

On a dit dans bien des livres que l'intérieur de Constantinople était laid et infect; on a eu tort: chaque coin de rue a son paysage, chaque maison ses merveilles; ceux qui se sont plaints n'ont point eu le courage d'affronter les innombrables chemins de Stamboul. Des trottoirs ne longent point les maisons, le gaz ne les éclaire pas, il est vrai ; mais où trouverezvous ces arbres de tous climats, ces pèlerins de toutes nations, ces souvenirs de tous les âges, et ce ciel, ce grand ciel bleu où volent les cigognes, les milans et les éperviers?

Quant à Flaubert, conquis, il écrit le 14 novembre 1850 :

C'est réellement énorme comme humanité. Ce sentiment d'écrasement que tu as éprouvé à ton entrée à Paris, c'est ici qu'il vous pénètre, en coudoyant tant d'hommes inconnus, depuis le Persan et l'Indien jusqu'à l'Américain et l'Anglais, tant d'individualités séparées dont l'addition formidable aplatit la vôtre. Et puis, c'est immense. On est perdu dans les rues, on ne voit ni le commencement ni la fin. Les cimetières sont des forêts au milieu de la ville. Du haut de la tour de Galata, on voit toutes les maisons et toutes les mosquées (à côté et parmi le Bosphore et la Corned'Or pleins de vaisseaux). Les maisons peuvent être comparées aussi à des navires, ce qui fait une flotte immobile dont les minarets seraient les mâts des vaisseaux de haut bord [phrase un peu entortillée, passons].

Le caractère romantique des cimetières de Constantinople l'impressionne fortement :

Le cimetière oriental est une des belles choses de l'Orient. II n'a pas ce caractère profondément agaçant que je trouve chez nous à ce genre d'établissement; point de mur, point de fossé, point de séparation ni de clôture quelconque. Ça se trouve à propos de rien, dans la campagne ou dans une ville, tout à coup et partout, comme la mort elle-même, à côté de la vie et sans qu'on y prenne garde. On traverse un cimetière comme on traverse un bazar. Toutes les tombes sont pareilles; elles ne diffèrent que par l'ancienneté. Seulement, à mesure qu'elles vieillissent, elles s'enfouissent et disparaissent, comme fait le souvenir qu'on a des morts. Les cyprès plantés en ces lieux sont gigantesques. Ça donne au site un jour vert plein de tranquillité. À propos de sites, c'est à Constantinople véritablement que l'on peut dire : un site! Ah! Quel tableau!

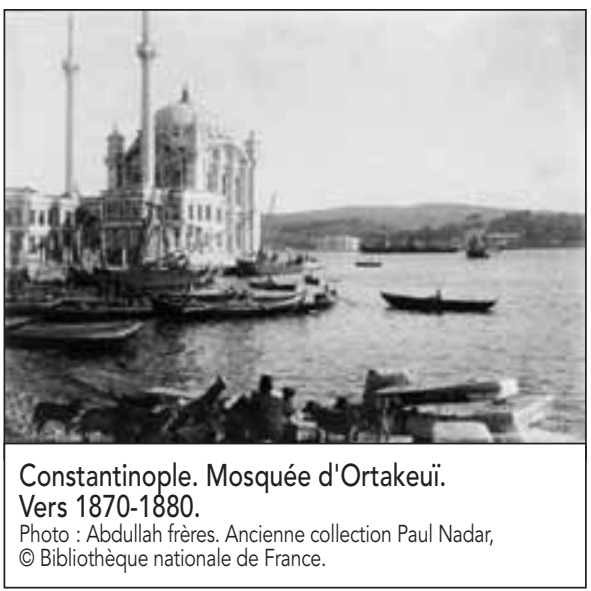

En 1852, sous la forme de feuilletons parus dans les journaux français de l'époque, Théophile Gauthier témoigne sur la Turquie. Cet écrivain ressent aussi un attrait pour les cimetières de Constantinople. Observant la non-séparation des morts et des vivants dans les cimetières, il s'exclame:

Peut-être les morts couchés sous les cyprès préfèrent-ils ce tumulte vivace au froid silence, à la morne solitude, à l'abandon glacial qui les isolent ailleurs ; ils restent mêlés à leurs contemporains, à leurs amis, à leurs descendants, et ne sont pas relégués en dehors de la circulation comme des objets sinistres ou des épouvantails; la cité vivante ne les rejette pas de son sein avec horreur et dégoût ; cette familiarité qui semble impie au premier abord, et au fond plus tendre que notre réserve superstitieuse.

Cette cité apporte aux voyageurs tous les délices recherchés, les cafés, les bains, les bazars et les femmes. Ainsi, l'expérience du bain est inoubliable et Flaubert confie à sa mère en 1851 :

Nous avons été hier pour prendre un bain turc. On nous a dit qu'on ne chauffait les bains qu'après le carnaval. Cela te donne la mesure de Patras. Tout est à l'avenant. Comme douceur orientale, le bain turc est une chose que je regretterai. Rien ne délasse et ne nettoie comme ça.

Théophile Gauthier décrit dans le journal parisien La Presse du 18 décembre 1852 un de ces cafés stambouliotes:

C'est un vrai plaisir de prendre là une de ces petites tasses de café trouble qu'un 
jeune drôle aux grand yeux noirs vous apporte sur le bout des doigts dans un grand coquetier de filigrane d'argent ou de cuivre découpé à jour, après une longue course dans les rues si fatigantes de Constantinople, et cela vous rafraîchit plus que toutes les boissons glacées ; à la tasse de café est joint un verre d'eau, que les Turcs boivent avant et les Francs après.

Ainsi, les traditions culturelles de l'Orient ont un impact sur les voyageurs français séduits par tant de raffinement.

Les bazars enchantent le voyageur, qui, comme Théophile Gauthier, sont séduits par la profusion des denrées et de leurs arômes au détour des rues tortueuses :

Le grand Bazar, pour lui conserver le nom que les francs lui donnent, couvre un immense espace de terrain et forme comme une ville dans la ville, avec ses rues, ses ruelles, ses passages, ses carrefours, ses places, ses fontaines, inextricable labyrinthe où l'on a de la peine à se retrouver, même après plusieurs visites. Ce vaste espace est voûté, et le jour y tombe de ces petites coupoles [...] Une odeur pénétrante, composée des arômes de tous ces produits exotiques vous monte aux narines et vous enivre.

Enfin les femmes retiennent l'attention du voyageur français, car il sait, si l'on en croit Théophile Gauthier, que « la première question que l'on adresse à tout voyageur qui revient d'Orient" est: "Et les femmes ? (Gauthier, 1990).

De nombreuses descriptions parsèment les récits des voyageurs écrivains, tantôt les femmes de leurs hôtes ainsi que leurs servantes, tantôt les femmes rencontrées lors de certaines escapades dans les maisons closes, ou bien les femmes du Harem.

Nerval décrit ce lieu clos de la maison dans lequel il y a bien une maîtresse parmi toutes les femmes: "cette dernière est la mère ou la belle-mère, ou l'épouse la plus ancienne ou celle qui a donné le jour à l'aîné des enfants ».

Si Flaubert aime donner des détails sur ses "exploits» sexuels, Gauthier brosse de beaux portraits de femmes turques ou d'au- tres origines comme les Arméniennes ou les Circassiennes. Ainsi, la ville de Constantinople est aussi un moyen pour lui de décrire des beautés exotiques richement vêtues :

\section{La Khanoun était somptueusement} parée, comme le sont chez elles les dames turques, surtout lorsqu'elles attendent quelque visite. Ses cheveux noirs divisés en une infinité de petites nattes, lui tombaient sur les épaules et le long des joues. Le sommet de la tête étincelait comme coiffée d'un casque de diamants formé par les quadruples chaînettes d'une rivière et par les pierres d'une eau admirable cousues sur une petite calotte en satin bleu-deciel qu'elles recouvraient presque entièrement.

Dans l'ensemble de ces relations de voyages des écrivains orientalistes français du XIX siècle, l'attrait des villes de cet Orient ne fait aucun doute. Ces jeunes bourgeois partent avec l'intention de découvrir des lieux mythiques que sont les villes célébrées et façonnées en grande partie par leurs lectures méticuleuses des précurseurs écrivains voyageurs et par les histoires racontées par leurs amis, soit au retour en France, soit dans des lettres envoyées par les uns et les autres. Ainsi, Maxime du Camp avait décrit à son ami Flaubert les voluptés de l'Orient lors de son voyage à Constantinople en 1844 ; quatre ans plus tard, il lui avait même dédié son livre Souvenirs et Paysages d'Orient. Une fois sur place, ces villes ne procurent pas le même bonheur à chacun des voyageurs et il importe de souligner que le désenchantement du moment fait partie des symptômes du voyageur qui a des attentes trop importantes, comme c'est souvent le cas chez Flaubert. La désillusion est d'autant plus grande que la réalité est loin de la vision imaginaire. Aussi le dépit s'installe-t-il.

Cependant tous ces écrivains sont restés marqués à vie par des expériences inoubliables, car elles portent en elles l'aventure intérieure. Plus que l'Orient géographique, ces hommes et parfois ces femmes apprennent à se découvrir face à l'Autre dans des lieux différents de leur ordinaire. Cette extraterritorialité les amène à reconquérir et même à reconstruire leur intériorité. Leurs œuvres postérieures ont bénéficié de ce travail sur le Soi et l'Autre pour le grand plaisir de leurs lecteurs.

\section{Conclusion}

La ville orientale est finalement un prétexte, une étape incontournable de la réalisation de ce parcours littéraire qui transforme l'homme en écrivain, car l'orientaliste français est cet occidental qui cherche son inspiration en Orient. La ville fait partie des escales de son périple initiatique, ce «Grand Tour» demeuré dans toutes les mémoires occidentales. Si l'on en croit Edward Said, "L'Orient est une création de l'Occident, son double, son contraire, l'incarnation de ses craintes et de son sentiment de supériorité tout à la fois, la chair d'un corps dont il ne voudrait être que l'esprit. »

Les écrivains voyageurs français sont à la recherche d'un fantasme, d'une «idéalisation poétique ", celle de la volupté et de la somptuosité des couleurs, mais aussi des valeurs perdues de l'Europe. Comme le fait remarquer Georges Corm dans son ouvrage Orient-Occident, la fracture imaginaire, "L'image d'une Europe qui a perdu son âme dans la course au progrès matériel va ainsi commencer à se cristalliser. L'idéalisation de l'Orient, de sa mystique, de son sens de l'honneur, de son mépris supposé des valeurs matérielles va se mettre en place. " Mais, en même temps, la supériorité de l'occidental, de l'écrivain français, ressurgit par un phénomène bien connu des immigrés et des exilés, celui de la "réidéalisation » du pays d'origine et de la dépréciation furieuse de l'oriental perçu comme un fourbe et un barbare.

En définitive, la ville orientale rêvée reste un sujet littéraire qui, peu à peu au tout début du XXe siècle, avec Loti ou Nadar, devient photographique. La photographie, par sa fonction d'authentification de l'expérience du voyageur, en introduisant l'image, témoigne du passage effectif du visiteur dans cet Ailleurs lointain. Ces villes sont alors appropriées par le voyageur devenu écrivain-photographe, à l'instar de Maxime du Camp, le précurseur en $1852^{6}$.

Chercheure invitée à la Chaire des Amériques de Rennes (France), Martine Geronimi est professeure associée au Département de géographie de l'UQAM. 


\section{Notes}

1 Les Mille et Une Nuits. Histoire du roi Shahriyar et de son frère le roi Schazaman : le sultan, Shéhérazade et Dinarzade. Illustration de Léon Carré (1878-1942). Ed. Piazza, Paris 1929-1932, 12 vol.

2 Les Cook's Eastern Tours sont disponibles peu avant l'ouverture du canal du Suez en 1869.

3 Le voyage en Palestine connaîtra dès lors un succès important.

4 Discours de Talleyrand à l'Institut de Paris, 3 juillet 1797. La décision d'intervenir en Égypte est prise le 16 mars 1798.

5 Gustave Flaubert, Correspondance 1 -18301851, Lettres à Louis Bouilhet, 20 août 1850.

6 Voir le site Web de la bibliothèque nationale de France, [http://expositions.bnf.fr/veo/orient _photo/index.htm].

\section{Bibliographie}

Amirou, Rachid (1995), Imaginaire touristique et sociabilités du voyage, Paris, Presses universitaires de France.

Berchet, Jean-Claude (1985), Le Voyage en Orient, anthologie des voyageurs français au XIXe siècle, Paris, Bouquins, Robert Laffont.

Bibliothèque nationale de France (BNF), Exposition consacrée au Voyage en Orient, [http://expositions.bnf.fr/veo/index.htm], (consulté les 25-26 février 2006).

Chateaubriand, René (1969), Itinéraire de Paris à Jérusalem, Paris, La Pléiade Gallimard.

Corm, Georges (2005), Orient-Occident: La fracture imaginaire, Paris, Éditions La découverte.

Flaubert, Gustave (1973), Correspondance 1. 1830-1851, Paris, La Pléiade Gallimard.
Flaubert, Gustave (1991), Voyage en Égypte, Paris Grasset.

Gauthier, Théophile (1990), Constantinople et autres textes sur la Turquie, Paris, La boîte à documents.

Hentsch, Thierry (1988), L'Orient imaginaire, Paris, Éditions de Minuit.

Lamartine de, Alphonse (2000), Voyage en Orient, Paris, Honoré Champion Éditeur.

Moussa, Sarga (2004), Le Voyage en Égypte. Anthologie des voyageurs européens de Bonaparte à l'occupation anglaise, Paris, Robert Laffont.

Nerval de, Gérard (1980), Voyage en Orient, Paris, Flammarion.

\section{TÉQROS}

TÉOROS invite tous les chercheurs et tous les professionnels qui œuvrent dans le domaine du tourisme, ou qui s'intéressent au tourisme sous tous ses aspects, à soumettre des articles de nature analytique à la revue. On peut soumettre un article en l'envoyant à :

\section{Bruno Sarrasin}

Directeur et rédacteur en chef

TÉOROS

Département d'études urbaines et touristiques

Université du Québec à Montréal

C.P. 8888, succ. Centre-Ville

Montréal, Québec, Canada

H3C 3 P8

Courriel : teoros@uqam.ca

Tél. : (514) 987-3000, poste 6959

Publiée trois fois l'an depuis 1982, TÉOROS, revue internationale de recherche en tourisme, se veut un outil intellectuel et professionnel pour les acteurs voués au développement du tourisme. Les problématiques liées aux rapports entre le tourisme et la culture ou entre le tourisme et la société, à la gestion ou à la planification en tourisme, à des cas particuliers de mise en tourisme ainsi qu'aux questions méthodologiques d'actualité, par exemple, comptent parmi les sujets abordés. Téoros souhaite étendre le champ de ces investigations en lançant un appel élargi à ses lecteurs pour constituer, dans chacun de ses numéros, mais indépendamment de la thématique adoptée, un regroupement de tels articles de nature analytique.

Les textes soumis doivent apporter une contribution scientifique originale, que ce soit par le biais d'information factuelle jusqu'alors inconnue ou par une nouvelle interprétation d'un thème particulier. Téoros vise avant tout le transfert des connaissances; son objectif est donc de promouvoir une meilleure compréhension des phénomènes liés au tourisme auprès d'un lectorat élargi.

Les auteurs doivent faire parvenir un manuscrit présenté selon les règles de la revue, que l'on peut consulter à www.teoros.uqam.ca. Habituellement, un article analytique compte environ 4000 mots et excè- de rarement 6000 mots, avec deux illustrations; on pourra cependant considérer des textes plus longs ou plus courts.

Les articles peuvent être soumis en anglais ou en français et doivent être accompagnés d'un résumé de 100 à 200 mots, dans la langue de l'article.

Les auteurs qui n'ont pas accès au site Internet de la revue peuvent contacter la rédaction pour obtenir copie des règles de présentation qu'ils devront suivre, quant aux références, notamment. Le manuscrit doit être fourni sur support informatique en format RTF.

Tous les manuscrits seront évalués par un comité de lecture qui décide des articles qui seront publiés. Le comité peut faire des suggestions ou demander des modifications. La rédaction transmettra l'avis du comité aux auteurs et s'assurera que les modifications demandées seront apportées. La réponse du comité est normalement donnée dans les trois mois suivant la soumission d'un article. 